\title{
Seizure Freedom after Lamotrigine Rash: A Peculiar Phenomenon in Epilepsy
}

\author{
Yosuke Kakisaka $^{1,2}$, Kazutaka Jin ${ }^{1}$, Kazuhiro Kato ${ }^{3}$, \\ Masaki Iwasaki ${ }^{4}$ and Nobukazu Nakasato ${ }^{1}$
}

\begin{abstract}
A 57-year-old right-handed woman with a history of left frontal lobe stroke had experienced episodes of language-expression difficulty followed by paraphasia lasting for approximately 30 seconds two years earlier. She was diagnosed with left frontal lobe epilepsy, and a lamotrigine regimen was initiated. This treatment had to be stopped five weeks after initiation because she developed a rash, and her drug lymphocyte stimulation test result was positive. Interestingly, she has since remained seizure free without requiring any antiepileptic medications. This adult case with a peculiar clinical course provides support for the hypothesis of immunomodulation process involvement in epilepsy, a phenomenon that was previously mainly seen in pediatric patients.
\end{abstract}

Key words: seizure freedom, lamotrigine rash, immune system, epilepsy

(Intern Med 53: 2521-2522, 2014)

(DOI: 10.2169/internalmedicine.53.2284)

\section{Introduction}

The peculiar phenomenon of seizure remission after some types of immunomodulation (e.g., viral infection) has been reported in pediatric patients (1). We herein describe a case of an adult epilepsy patient who experienced seizure remission without additional antiepileptic medication after an immunomodulation process that manifested as a rash and was confirmed by a positive drug lymphocyte stimulation test (DLST) for lamotrigine (LTG).

\section{Case Report}

A 57-year-old right-handed woman had been healthy until age 51 when she suffered a stroke involving the left frontal lobe. A tissue-type plasminogen activator was administered, but she subsequently exhibited mild right hemiparesis. She started to experience episodes of language-expression difficulty followed by paraphasia lasting for approximately 30 seconds at age 55. The frequency of these episodes gradu- ally increased to two to three per day, and she was referred to our department at age 56. She was neurologically intact between these episodes. A blood examination did not reveal any abnormalities. Magnetic resonance imaging showed encephalomalacia in the medial part of the left frontal lobe (Figure). Electroencephalography did not reveal clear interictal spikes, and ictal recording was not possible. Based on the findings indicating left frontal lobe epilepsy, LTG administration was started with an initial dose of $25 \mathrm{mg}$ that was gradually and appropriately increased. She became seizure-free with $100 \mathrm{mg}$ of LTG, but this treatment had to be stopped five weeks after initiation because of a rash that appeared on all her extremities. The diagnosis was erythema multiforme minor, and none of her symptoms suggested drug-induced hypersensitivity syndrome. LTG administration was stopped based on the judgment that LTG was responsible for her rash, and her skin symptoms resolved after treatment cessation. Her positive DLST for LTG supported the diagnosis to explain her dermatological symptoms, although general blood examinations did not reveal any abnormalities. She has remained seizure-free for 18 months without requir-

\footnotetext{
${ }^{1}$ Department of Epileptology, Tohoku University School of Medicine, Japan, ${ }^{2}$ Department of Pediatrics, Tohoku University School of Medicine, Japan, ${ }^{3}$ Department of Neurology, Tohoku University School of Medicine, Japan and ${ }^{4}$ Department of Neurosurgery, Tohoku University School of Medicine, Japan

Received for publication December 9, 2013; Accepted for publication March 30, 2014

Correspondence to Dr. Kazutaka Jin, jink@med.tohoku.ac.jp
} 


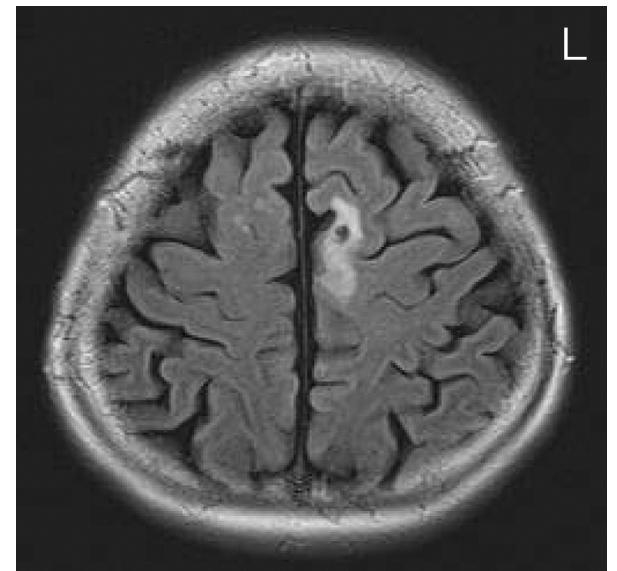

Figure. Fluid-attenuated inversion recovery magnetic resonance image showing a hyperintense lesion in the left medial frontal region indicating a previous infarction.

ing any antiepileptic medications.

\section{Discussion}

Some types of cytokines produced during viral infections, such as interleukin-10 and transforming growth factor beta, are considered to counteract inflammatory processes. Such anti-inflammatory cytokines may suppress immunopathological processes in a patient with epilepsy, leading to spontaneous seizure remission (2). The phenomenon of seizure remission after a viral infection could also be explained by several mechanisms (3). First, some viruses directly suppress epileptic seizures in the brain. Second, increased antiepileptic drugs (AEDs) levels caused by dehydration following viral infection suppress epileptic seizures. Third, some viruses induce the elevation of serum transaminase level, resulting in increased AEDs levels. Fourth, increased serum cortisol level following severe infection suppresses epileptic seizures. Finally, the ketogenic effect of fasting during viral infection induces a seizure-free state. Various studies have investigated the relationship between the occurrence of epilepsy and the immune system (4-6), including profiling of circulating lymphocytes $(5,6)$. An examination of the immunological profiles of 24 patients with focal epilepsy and 30 of their first-degree relatives found that the epileptic group had significantly fewer circulating CD4 "helper" T cells and more CD8 "cytotoxic"/"suppressor" T cells than the control group, thus suggesting that focal epilepsy may be linked to immune dysregulation (6).

Based on these previous findings, we speculate that immunomodulation processes was the most probable mechanism underlying epilepsy remission in the present case, although we could not strictly exclude incidental cooccurrence of seizure remission and the appearance of the LTG rash. Our findings of an abnormal type IV reaction, in which the T cell system is involved, and the positive DLST result for LTG suggest that an abnormal immunological process was responsible for generating focal epilepsy in our patient, and this was modified by the immunological change manifesting as the LTG rash.

Unfortunately, because we only observed this peculiar phenomenon in a single patient, we therefore could not elucidate the mechanism. However, physicians should be more aware of this rare but possibly important phenomenon, and reports of additional cases might help to clarify the mechanism in the future.

The authors state that they have no Conflict of Interest (COI).

\section{References}

1. Fujita Y, Imai Y, Ishii W, et al. Improvement of intractable childhood epilepsy following acute viral infection. Brain Dev 33: 6268, 2001.

2. Hattori H. Spontaneous remission of spasms in West syndromeimplications of viral infection. Brain Dev 23: 705-707, 2001.

3. Yamamoto H, Yamano T, Niijima S, Kohyama J, Yamanouchi H. Spontaneous improvement of intractable epileptic seizures following acute viral infections. Brain Dev 26: 377-379, 2004.

4. Billiau AD, Wouters CH, Lagae LG. Epilepsy and the immune system: is there a link? Eur J Paediatr Neurol 9: 29-42, 2005.

5. Eeg-Olofsson O, Prchal JF, Andermann F. Abnormalities of Tlymphocyte subsets in epileptic patients. Acta Neurol Scand 72: 140-144, 1985.

6. Eeg-Olofsson O, Osterland CK, Guttmann RD. Immunological studies in focal epilepsy. Acta Neurol Scand 78: 358-368, 1988.

(C) 2014 The Japanese Society of Internal Medicine http://www.naika.or.jp/imonline/index.html 